Supporting Information

\title{
Nonlinear predictive control of a bioreactor by surrogate model approximation of flux balance analysis
}

Rafael D. de Oliveira†, Matheus N. Guedest, José O.A. Matias ${ }^{\ddagger}$, and Galo A. C. Le Roux*

†Department of Chemical Engineering Polytechnic School, University of São Paulo, São Paulo, Brazil

‡Department of Chemical Engineering, Norwegian University of Science and Technology (NTNU), Trondheim, Norway

E-mail: galoroux@usp.br 

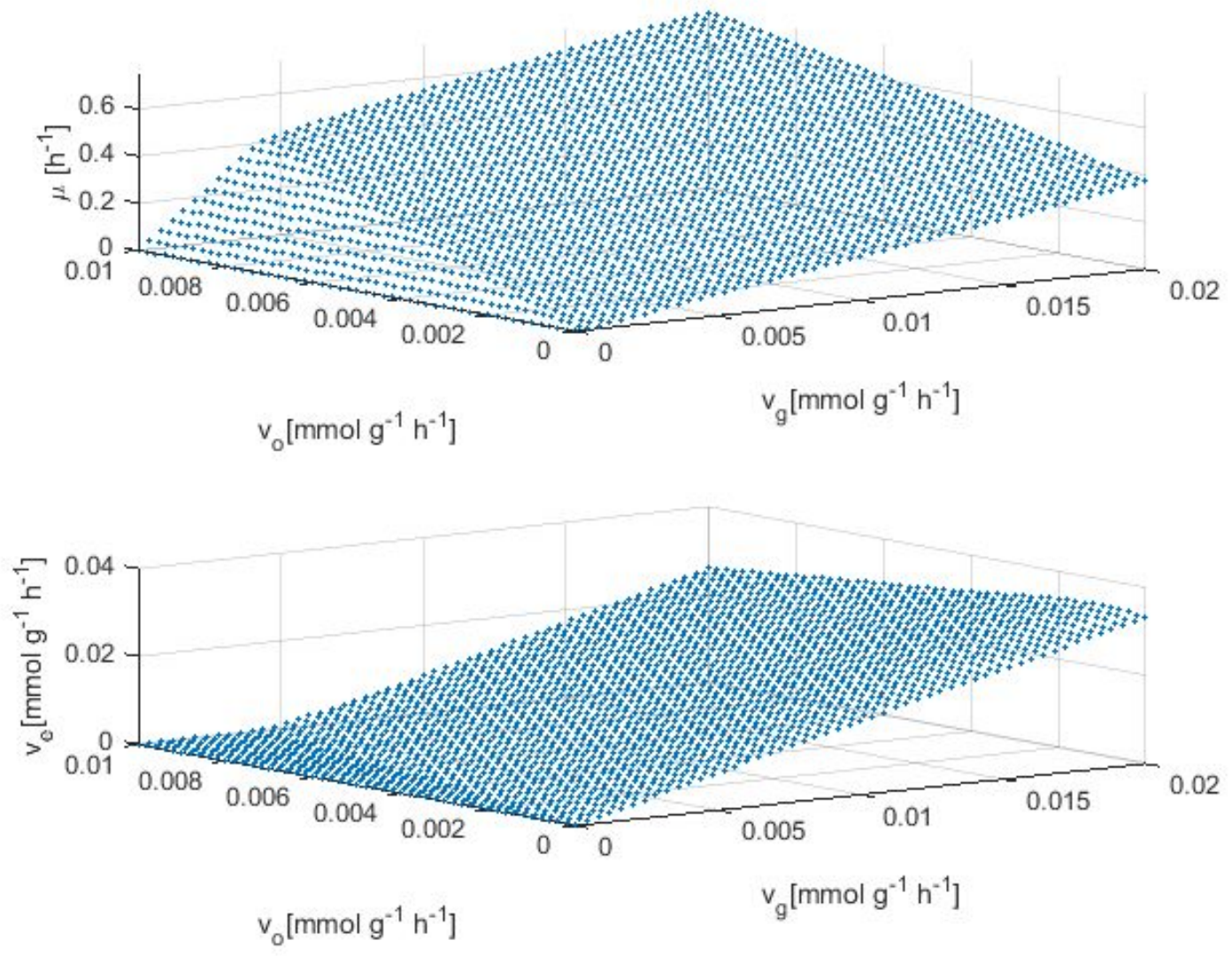

Figure S1- Profiles of the FBA solutions for different values of the uptake rates of glucose and of oxygen. We solved the FBA problem for every value of the independent variables in an equidistant 50 by 50 grid. Here, only one zone of operation was used. 

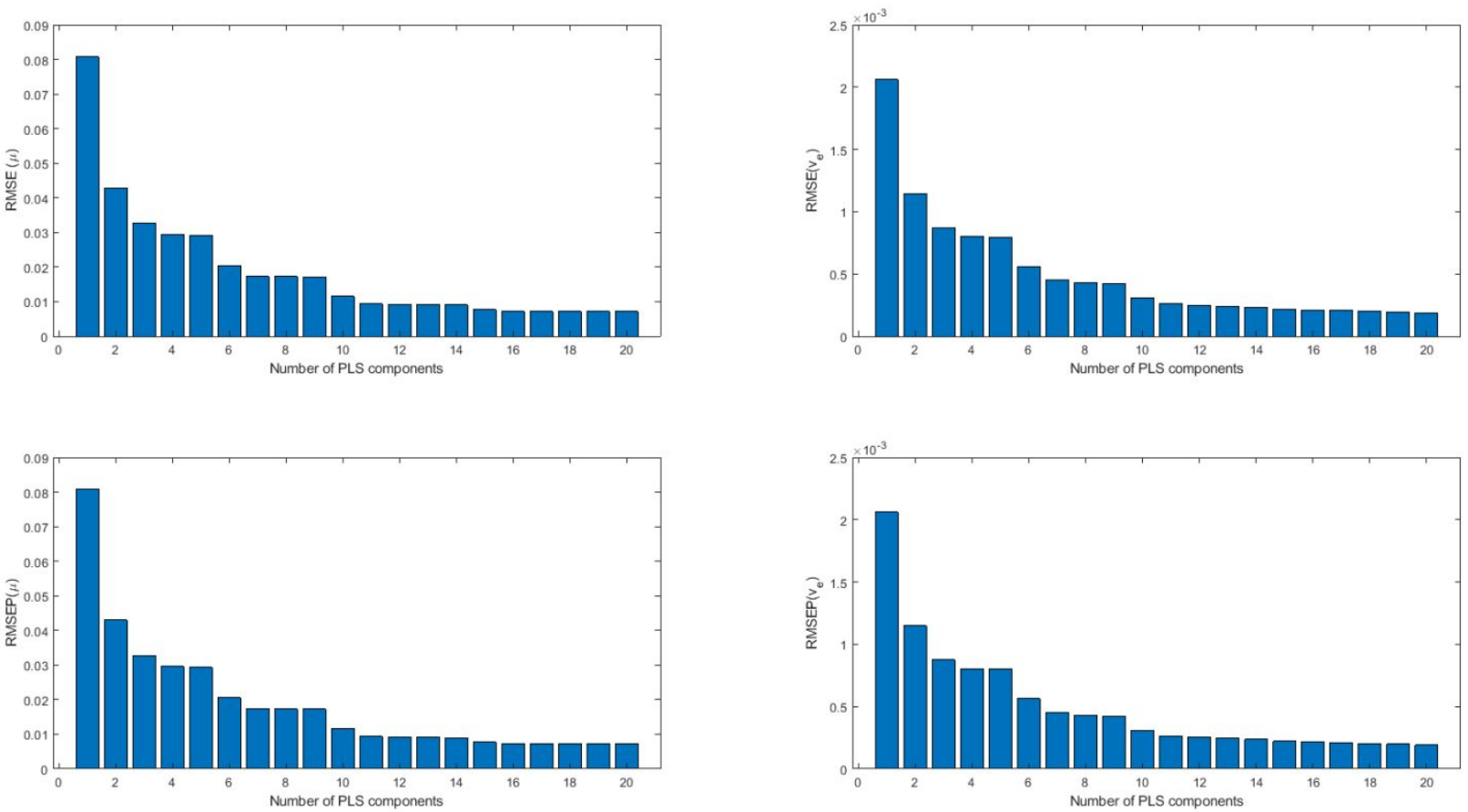

Figure S2- Relative RMSE and RMSEP for growth rate model and the exchange ethanol rate as a function of the number of PLS components for a surrogate model with a single zone (Figure S1). 

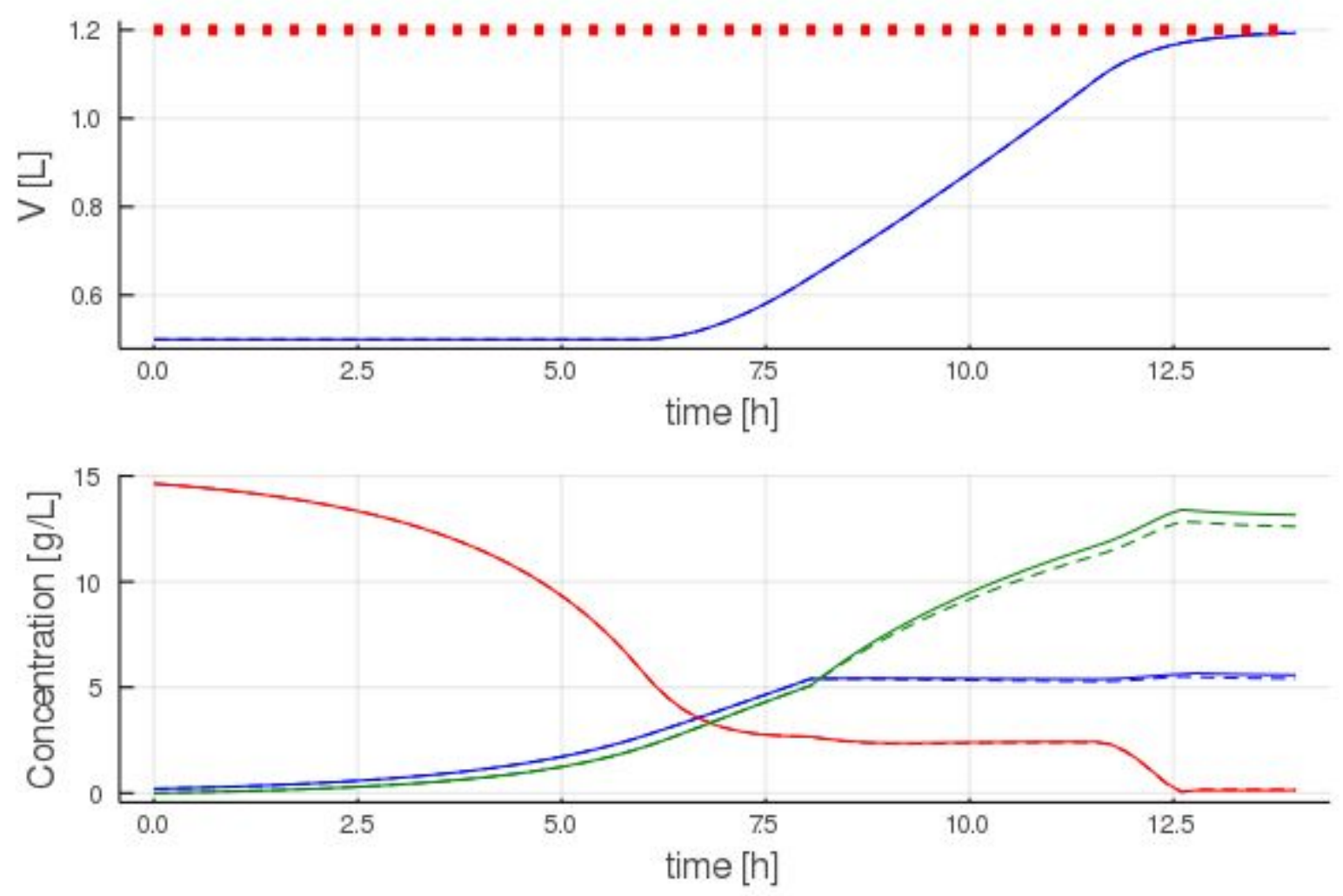

Figure S3 - Profiles obtained using the surrogate model with a single zone (--) and three zones (-) in the controller. The standard dFBA was used as the bioreactor model. The concentration profiles are glucose (red), biomass (blue) and ethanol (green). 

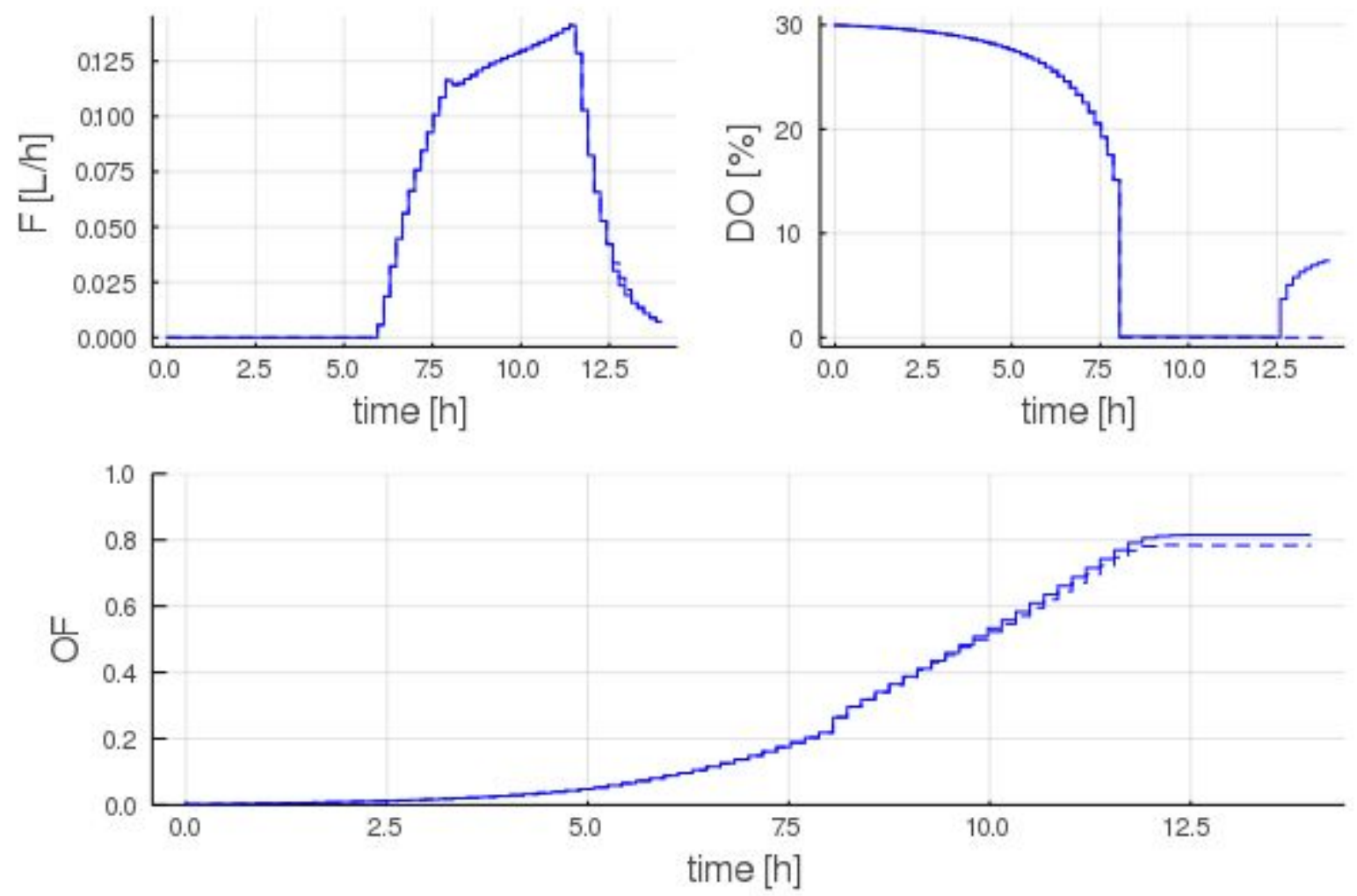

Figure S4 - Control variables and objective function (OF) profiles obtained using the surrogate model with a single zone (--) and three zones (-) in the controller. The standard dFBA was used as the bioreactor model. 

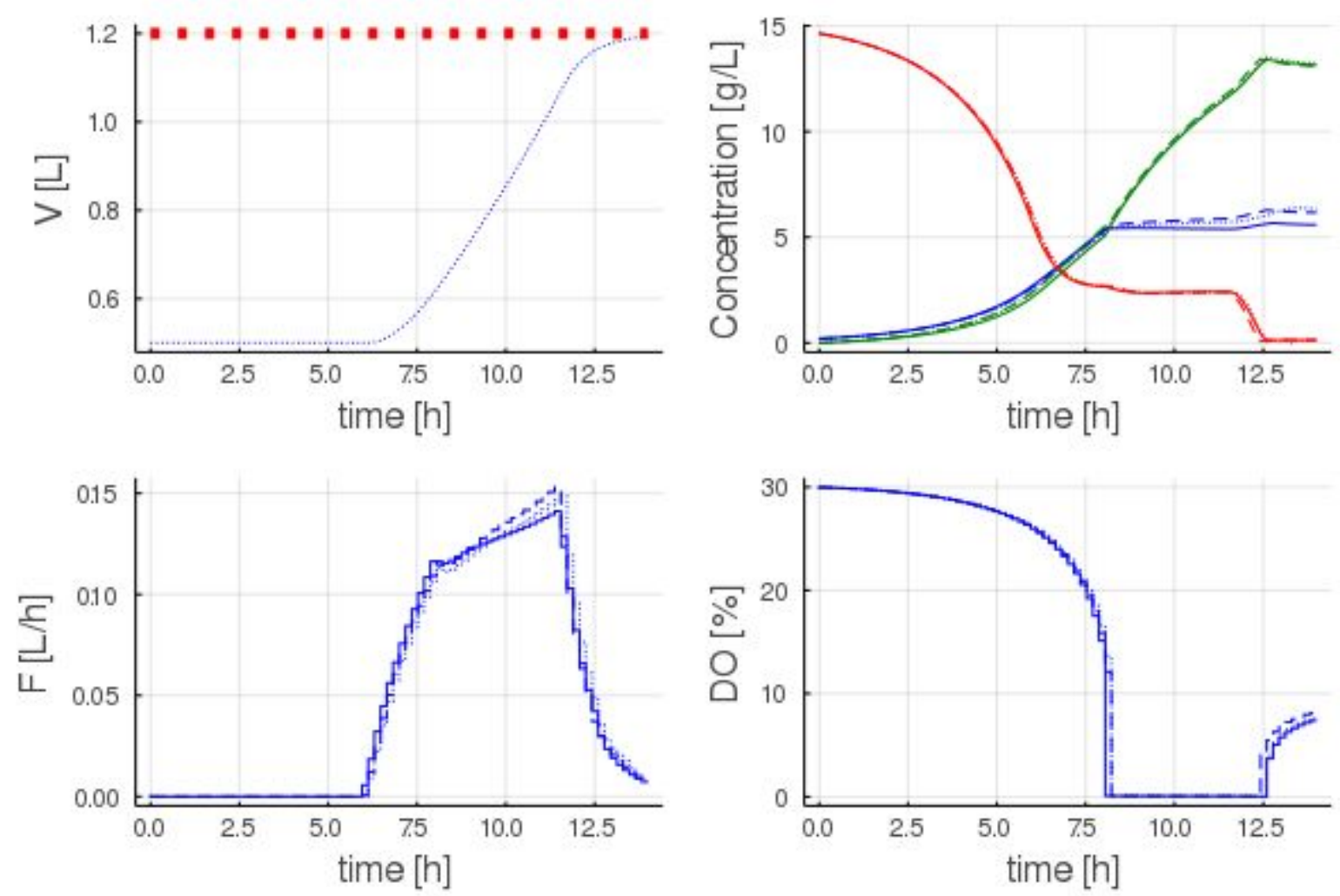

Figure S5- Comparison between different GSM for the plant-model: Yeast 8.3 (-), iND750 (--) and iMM904 ( ‥). The simulations were performed on open-loop operation. The concentration profiles are glucose (red), biomass (blue) and ethanol (green). 